\title{
Small Bowel Neoplasias: Current Options for Diagnosis, Staging and Therapeutic Management
}

\author{
Lucia C. Fry a, c Juan Pablo Gutierrez ${ }^{c}$ Ivan Jovanovic ${ }^{b}$ \\ Klaus Mönkemüller ${ }^{c}$ \\ a Department of Internal Medicine, Gastroenterology and Infectious Diseases, \\ Marienhospital Bottrop, Bottrop, Germany; ${ }^{b}$ Clinic for Gastroenterology and Hepatology, \\ Clinical Center of Serbia, Belgrade, Serbia; ' Division of Gastroenterology and Hepatology, \\ Basil Hirschowitz Endoscopic Center of Excellence, Birmingham, Ala., USA
}

\section{Key Message}

New imaging techniques such as capsule endoscopy and balloon-assisted endoscopy have facilitated the early diagnosis of small bowel malignancies, leading to a better understanding of the biology of these tumors and to improved clinical outcomes for the patient.

\section{Practical Implications}

Although relatively rare, the incidence of tumors and polyps in the small intestine has increased over the past 20 years. The use of endoscopic or radiologic techniques is crucial for the detection and resection of polyps. It is highly recommended to perform a submucosal injection of epinephrine-saline solution prior to endoscopic resection. Due to their non-specific symptoms, neuroendocrine tumors are often diagnosed in advanced stages; surgical excision of the primary tumor is key to a better prognosis. The main therapies for neuroendocrine tumors are surgery, chemoembolization, chemotherapy and the use of somatostatin analogues. Small bowel adenocarcinomas are extremely rare but can be highly fatal. Surgical resection is still the core treatment, though fluoropyrimidine and oxaliplatin-based chemotherapy have shown beneficial effects for the treatment of metastatic disease. Amongst the gastrointestinal stromal tumors, 30\% are located in the small intestine. The management of gastrointestinal stromal tumors requires a combination of surgery, pathology techniques and pharmacological interventions, including the use of tyrosine kinase inhibitors. 
Fry et al.: Small Bowel Neoplasias: Current Options for Diagnosis, Staging and Therapeutic Management

\title{
Key Words
}

Gastrointestinal stromal tumor · Neuroendocrine tumor · Peutz-Jeghers syndrome · Small bowel adenocarcinoma $\cdot$ Small bowel polyps

\begin{abstract}
Although small bowel polyps and tumors are rare, their incidence has increased significantly over the past 30 years. Small bowel malignancies can be classified depending upon their cellular origin into four principal histologic types: adenocarcinomas, lymphomas, neuroendocrine tumors or carcinoids and lymphomas, which also include gastrointestinal stromal tumors. The relative 'rarity' of these tumors has led to stagnation in the development of effective curative or adjuvant therapies. Thus, the prognosis of most of these tumors is still dismal. Nevertheless, hope is now on the horizon as new methods such as capsule endoscopy and balloon-assisted enteroscopy have contributed to a rise in the diagnosis of these lesions and a diagnosis at earlier stages. Using balloon-assisted enteroscopy methods it is possible to resect most small bowel polyps such as adenomas and hamartomas. Improved imaging methods have led to a better understanding of these pathologies and hopefully will bring new hopes in therapy. In addition, multi-center studies are being performed to determine the best therapeutic options for small bowel tumors.

(C) 2013 S. Karger AG, Base
\end{abstract}

\section{Introduction}

Although the mucosa of the small intestine encompasses about $90 \%$ of the luminal surface area of the digestive system, small bowel polyps and tumors are relatively rare [1]. Nevertheless, the incidence of small bowel neoplasms has increased significantly over the past 20 years [2-4]. This increased incidence has been paralleled by the advent of capsule endoscopy and balloon-assisted enteroscopy [5-8]. We and others have shown that the incidence of polyps and tumors in patients undergoing deep enteroscopy ranges from 5 to $17 \%$ [5, 9]. In a large single-center study from Germany, our group found that the incidence of small bowel polyps and tumors in 301 patients undergoing 401 double balloon enteroscopies was 9.6\% [5].

Small bowel malignancies can be classified depending upon their cellular origin into four principal histologic types: adenocarcinomas, lymphomas, neuroendocrine tumors (NETs) or carcinoids and lymphomas, which also include gastrointestinal stromal tumors (GISTs) [10]. More common than malignant tumors are small bowel polyps [5]. The most common small bowel polyps are adenomas in familial adenomatous polyposis (FAP) syndrome, hamartomas (Peutz-Jeghers and Cronkhite-Canada syndromes). Table 1 lists a vast differential diagnosis of small bowel polyps and tumors. This review will focus on small bowel polyps, adenocarcinomas, NETs and GISTs. Lymphomas are not addressed in this review.

\section{Small Bowel Polyps}

Small bowel polyps may be subdivided into several types, such as hyperplastic polyps, lipomas, sporadic adenomas, FAP, familial juvenile polyposis, and those associated with Peutz-Jeghers syndrome (table 1, fig. 1). Gastrointestinal polyposis syndromes are defined by the presence of multiple polypoid lesions throughout the gastrointestinal tract $[11,12]$. These polyposis syndromes can be broadly classified as inherited or non-inherited (table 1). These syndromes can also be classified according to the histologic characteristics of polyps into adenomatous or hamartomatous polyposis syndromes. Patients with hereditary polyposis 


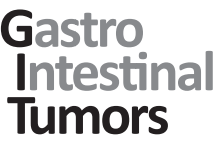

Table 1. Small bowel polyps and tumors

\begin{tabular}{l|l}
\hline Gastrointest Tumors 2014;1:9-17 \\
\hline DOI: 10.1159/000355210 & $\begin{array}{l}\text { @ 2013 S. Karger AG, Basel } \\
\text { www.karger.com/gat }\end{array}$ \\
\hline
\end{tabular}

Fry et al.: Small Bowel Neoplasias: Current Options for Diagnosis, Staging and Therapeutic Management

\begin{tabular}{ll}
\hline Malignant tumors & \\
\hline Primary tumors & Metastatic tumors \\
NET & Lung adenocarcinoma \\
Adenocarcinoma & Squamous cell carcinoma \\
GIST & Small cell carcinoma \\
B cell lymphoma & Large cell carcinoma \\
Follicular lymphoma & Breast carcinoma \\
Mantel cell lymphoma & Lobular \\
Diffuse large B cell lymphoma & Ductal \\
Burkitt-like lymphoma & Melanoma \\
Burkitt lymphoma & Amelanotic melanoma \\
T cell lymphoma & Ovarian adenocarcinoma \\
Enteropathy-type T cell lymphoma & Carcinosarcoma \\
Anaplastic lymphoma & Gastric adenocarcinoma \\
Peripheral T cell lymphoma & Colon adenocarcinoma \\
Natural killer cell lymphoma & Gallbladder adenocarcinoma \\
Sarcoma & Renal cell carcinoma \\
Leiomyosarcoma & Pelvic adenocarcinoma \\
Histiocytic sarcoma & Pancreatic carcinoma \\
Ewing's sarcoma & Osteosarcoma \\
& Choriocarcinoma \\
\hline Benign polyps and tumors & Testicular germ cell tumor \\
\hline Adenoma & \\
Lipoma & Hemangioendothelioma \\
Hamartoma & Lymphangioma \\
\hline & Lymphatic cyst \\
\hline Abmangioma & Myxoma \\
\hline & \\
\hline &
\end{tabular}

have an increased risk of malignancies, up to a $100 \%$ risk of colorectal cancer in patients with FAP [11]. The risk of extracolonic (i.e. small bowel) malignancies varies according to the syndrome. Classically it has been thought that only adenomatous polyps present a risk of malignant transformation, but newer data also show that hamartomatous polyps have foci of adenoma, and these have also been associated with an increased risk of cancer development [11]. Patients with Cronkhite-Canada syndrome are also at increased risk of developing small bowel cancer [6]. Patients with FAP are at increased risk of developing duodenal, ampullary and jejunal adenocarcinomas [11]. The overall risk of small bowel cancer in FAP is 4-12\%, or up to 300-fold higher than in the general population [2, 7-9]. Saurin et al. [13] have described that patients with mutations in the central part of the APC gene have more severe duodenal polyposis. Genotypic-phenotypic characterizations to correlate with duodenal polyposis and jejunal severity may also aid in determining screening methods (esophagogastroduodenoscopy and duodenoscopy versus double balloon enteroscopy or capsule endoscopy) and surveillance intervals of these patients [14]. In addition, we believe that searching for polyps beyond the duodenum in FAP is important because of the reported occurrence of jejunal adenocarcinoma in these patients [11].

\section{Surveillance in Polyposis Syndromes}

Surveillance of patients with adenomatous and hamartomatous polyposis syndromes is important to detect and resect polyps in the gastrointestinal tract in order to decrease the incidence of cancer, but also to avoid complications such as bleeding, obstruction and intussusception [11]. Surveillance methods include capsule endoscopy, balloon-assisted enter- 

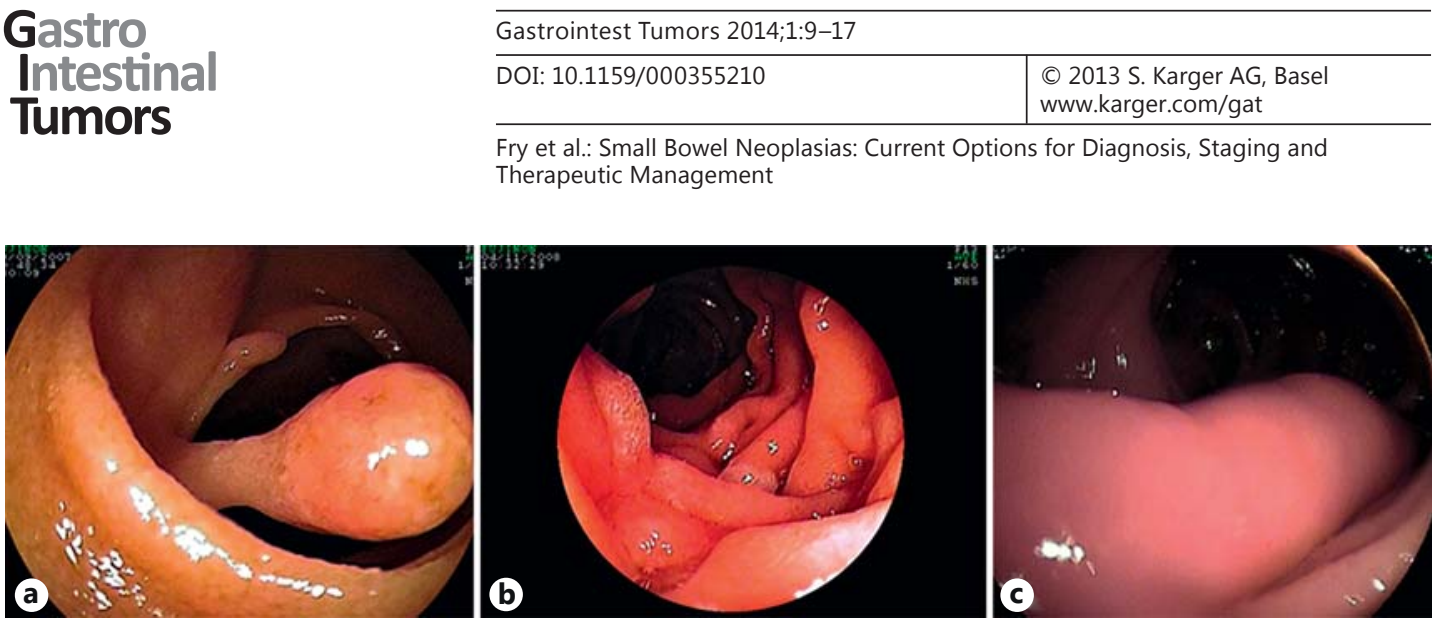

Fig. 1. Small bowel polyps. a Hamartoma in Peutz-Jeghers syndrome. b Adenoma in FAP syndrome. c Jejunal lipoma.
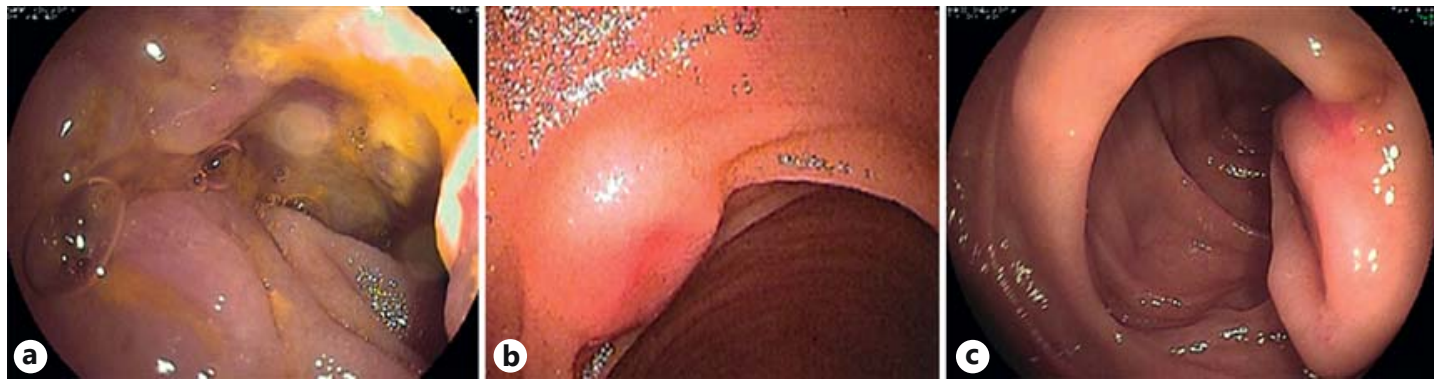

Fig. 2. Small bowel tumors. a Adenocarcinoma of the jejunum. b NET of the ileum. c Typical GIST with large groove in the center.

oscopy, push enteroscopy, intraoperative endoscopy, or radiologic techniques such as enteroclysis, computed tomography (CT) enteroclysis or magnetic resonance enteroclysis. Capsule endoscopy has proved to be useful for the evaluation of the small bowel in patients with polyposis syndromes, also when compared to other imaging methods such as magnetic resonance imaging, CT enterography and push enteroscopy [11].

\section{Therapy of Small Bowel Polyps}

The issue of resective or ablative therapies in patients with familial polyposis syndromes is gaining increasing importance. Most polyps in patients with Peutz-Jeghers syndrome and FAP can be removed endoscopically $[11,12]$. We want to emphasize that careful attention must be paid to the endoscopic removal technique as there are some reported and many unreported cases of perforation. Removal of polyps is performed using either snare polypectomy or mucosectomy techniques. When dealing with larger polyps with thick stalks and flat lesions, we always proceed to endoscopic resection by submucosal injection of epinephrine-saline solution $(1: 20,000$ to $1: 100,000)$ ('submucosal cushion'). The submucosal cushion is an essential technique when performing advanced polypectomy within the luminal gastrointestinal tract because the small bowel has a very thin wall and the submucosal cushion is especially suited for resection of these mucosal lesions. The submucosal cushion basically entails the elevation of the target lesion as a result of submucosal injection of various liquid substances [12]. By raising the polyp from the submucosa, the endoscopic resection is facilitated and a more complete resection of the neoplastic tissue can be achieved. In addition, by lifting the submucosa from the deeper layers of the gut wall, the depth of injury to the gut wall 
is decreased by increasing the distance between the burn and the muscularis propria and serosa, which in turn may result in fewer complications [12]. For small bowel polypectomy we recommend to use a submucosal cushion whenever possible, especially for all flat lesions, polyps with thick pedicles and any polyp $>10 \mathrm{~mm}$ in maximal length.

\section{Small Bowel Tumors}

Though it comprises $3 / 4$ of the length and $>90 \%$ of the surface area of the gastrointestinal tract, the small bowel harbors relatively few primary neoplasms (which account for about $5 \%$ of all gastrointestinal tumors) and fewer than $2 \%$ of gastrointestinal malignancies. Nevertheless, recent data show that the incidence is rising. In a large United States study, Haselkorn et al. [3] analyzed the four histologic types of small bowel cancer, adenocarcinomas, carcinoid tumors, lymphomas and sarcomas. The authors found that men had higher rates than women for all types of small bowel cancer. Blacks had almost twice the incidence of carcinomas and carcinoid tumors compared to whites (10.6 vs. 5.6 per million people and 9.2 vs. 5.4 per million people, respectively). They showed that the incidence of small bowel cancer has risen, with the greatest increase for carcinoid tumors (21\%) and black men $(120 \%)$. Interestingly a geographic correlation between the incidence of small and large bowel cancer, but not of small bowel and stomach cancer, was observed, which suggests shared etiologies. A rise in incidence of small bowel cancers has also been documented by Severson et al. [4]. They found that rates increased most dramatically in black males, with 2and 4-fold increases in adenocarcinomas and carcinoid tumors, respectively. The only rates that remained relatively unchanged were those of adenocarcinoma among white females. It remains to be determined whether changing environmental factors are important causes of these observed trends.

Small bowel tumors typically are asymptomatic in their early stages, but more than $90 \%$ of patients eventually develop symptoms as the disease progresses. No hallmark presentations can be observed in small bowel tumors; possible signs and symptoms mainly include abdominal pain, obscure gastrointestinal bleeding, obstruction, volvulus, diarrhea and/or constipation.

\section{NETs of the Small Bowel}

NETs arise from the enterochromaffin cells at the base of the crypts of Lieberkühn and account for about one third of all small bowel neoplasms. Small bowel carcinoids begin as small polypoid masses, but have a tendency to invade into the submucosa and eventually infiltrate the muscularis propria, serosa, and then invade mesenteric lymph nodes [15]. Even small NETs can metastasize to the lymph nodes and liver $[6,15]$. Due to their endocrine function they may induce symptoms such as diarrhea, flushing and sweating. Gastrointestinal bleeding, obstruction or perforation derive from local complications at the site of primary tumor involvement in the small bowel [15]. Localized NETs are less frequent than metastatic ones; in fact, up to $75 \%$ of patients with small bowel NETs and $30-85 \%$ of patients with pancreatic NETs present with liver metastases either at the time of diagnosis or during the course of the disease. The predominant metastatic site is the liver, which is the best prognostic marker of survival regardless of the primary site [16].

Because of its non-specific initial symptoms, patients are often diagnosed with a NET only in advanced, metastasized stages of the disease, when a curative therapeutic option is beyond practicability $[6,15]$. The search for the primary tumor is often cumbersome but of 
Fry et al.: Small Bowel Neoplasias: Current Options for Diagnosis, Staging and Therapeutic Management

crucial importance, as its surgical excision leads to a better prognosis even in a metastasized NET $[15,16]$. Octreotide scintigraphy is an approved method with a high diagnostic yield for detecting also early stages of NET, but due to its two-dimensional view the differentiation between mesenteric and intestinal localization is not possible, and in case of low receptor density a NET might not be detectable [16]. However, current guidelines recommend abdominal ultrasound, contrast-enhanced CT or magnetic resonance imaging of the upper and lower abdomen as well as octreotide scintigraphy and small bowel enteroclysis for the detection of the primary tumor in the small bowel [16].

An elegant study using capsule endoscopy by van Tuyl et al. [6] reports a diagnostic yield of $45 \%$ ( 9 of 20 patients) for detecting NETs of the small bowel. Tumors $<2 \mathrm{~cm}$ in size are usually discovered incidentally. They appear as smooth, rounded, 1-2 cm elevations in the distal small bowel. The tumors are yellowish and may be mucosal or submucosal in appearance, without desmoplastic changes, and be indistinguishable from other tumors such as GISTs or lipomas. Despite their classic endoscopic characteristics, the study by van Tuyl et al. [6] clearly highlights the difficulty of luminal tumor search in NETs, as in 7 patients with positive octreotide scintigraphy capsule endoscopy was negative for a supposed mesenteric localization of the NET. Double balloon enteroscopy is not a good option in the search for NETs [15].

\section{Therapy of NETS}

The therapeutic options for NETs depend on the type, size and location of the primary tumor. Three main therapeutic axes have been individualized in the field of well-differentiated NETs (corresponding to grades 1 and 2 of the new WHO classification 2010): (1) antitumor activity of somatostatin analogues, particularly in slowly progressive metastatic NETs with limited hepatic invasion, (2) targeting angiogenesis in these hypervascular tumors, and (3) targeting the mTOR pathway involved in NET carcinogenesis. The key therapies for NETs are surgery, chemoembolization, chemotherapy and somatostatin analogues. If surgical resection or interventional therapies of the hepatic tumor burden are not feasible or if the metastases are not confined to the liver, systemic treatment remains the only option. Everolimus was beneficial for the treatment of small bowel NETs in one randomized trial. Patients with poorly differentiated NETs, regardless of the primary tumor localization, are candidates for cisplatin and etoposide chemotherapy regimens. Peptide receptor radionuclide therapy is reported to be an effective treatment option for patients with good performance status and high somatostatin receptor scintigraphy uptake as well as without major liver involvement [16].

\section{Adenocarcinoma of the Small Bowel}

Adenocarcinomas of the small bowel are rare, accounting for $1 \%$ of all gastrointestinal malignancies or about 2,000 cases/year in the United States [17]. Small bowel adenocarcinomas are still considered to be highly fatal due to their non-specific presentation at the time of diagnosis. In addition, until recently most patients with adenocarcinoma presented with advanced disease. However, this has markedly changed since the advent of better crosssectional imaging techniques, capsule endoscopy and balloon-assisted enteroscopy techniques $[5,8]$. These methods have allowed for earlier recognition of this aggressive cancer. Although frequently grouped under the same therapeutic umbrella as large bowel adenocarcinomas, small bowel adenocarcinomas are distinct clinical and molecular entities.

Adenocarcinoma is the most frequent primary small bowel tumor, accounting for $40 \%$ of these neoplasms [17]. More than half of small bowel adenocarcinomas arise in the duodenum, even though this organ comprises only $4 \%$ of the entire length of the small bowel. Most arise 
in the region of the ampulla of Vater. A smaller percentage of tumors arise in the jejunum, particularly in the first $30 \mathrm{~cm}$ distal to the ligament of Treitz. Ileal carcinomas are the least common, except in patients with Crohn's disease [18].

\section{Diagnosis and Staging of Small Bowel Adenocarcinomas}

On barium studies, advanced small bowel adenocarcinomas appear as 'apple core' lesions similar to those found elsewhere in the gastrointestinal tract. These annular lesions are short, circumferentially narrowed segments with mucosal ulceration and overhanging proximal and distal borders. These malignant strictures are usually rigid, with a fixed, unchanging appearance during compression. On CT, small bowel adenocarcinomas may manifest as a discrete tumor mass, annular narrowing with abrupt concentric or irregular 'overhanging edges' or an ulcerative lesion [18]. Endoscopic diagnosis of adenocarcinomas remains rare. However, since the advent of balloon-assisted enteroscopy we are increasingly diagnosing adenocarcinoma of the small bowel $[5,8,19]$. The classic endoscopic appearance is that of an ulcerated lesion (fig. 2a).

\section{Therapy of Small Bowel Adenocarcinomas}

Earlier detection has led to curative therapies, such as resection. Surgical resection remains the mainstay of therapy for locoregional disease. Modern chemotherapy regimens have produced improved response rates and survival rates when compared to historical controls. In the metastatic setting, fluoropyrimidine and oxaliplatin-based chemotherapy has shown clinical benefit in prospective non-randomized trials $[9,17]$. In a classic study Overman et al. [20] showed that chemotherapy with 5-fluorouracil and a platinum compound improves outcomes in metastatic small bowel adenocarcinoma. This study is important, as the authors were able to analyze the outcomes of 80 patients with metastatic small bowel cancer who were treated with chemotherapy at the University of Texas MD Anderson Cancer Center between 1978 and 2005. This study presents the largest number of patients with metastatic small bowel cancer treated with chemotherapy in the literature, and the results suggest that the combination of 5 -fluorouracil and a platinum compound leads to a higher response rate and progression-free survival compared with other chemotherapy regimens. The authors concluded that a prospective investigation of platinum analogues in the treatment of small bowel adenocarcinomas is warranted. Overman et al. [21] also studied the effect of adjuvant chemotherapy in small bowel adenocarcinoma. The authors identified 54 patients with small bowel adenocarcinoma who underwent margin-negative surgical resection and were evaluated after surgery. The authors found that the use of adjuvant therapy for curatively resected small bowel adenocarcinomas was associated with an improvement in diseasefree survival, but not in overall survival.

\section{GISTs}

GISTs are mesenchymal neoplasms that arise in the gastrointestinal tract, usually in the stomach or the small intestine, and rarely elsewhere in the abdomen. While most GISTs are located in the stomach, $30 \%$ of GISTs are found in the small bowel [18]. A characteristic of GISTs is their extraluminal growth [19, 22]. Small bowel GISTs tend to be more aggressive and have a worse prognosis. Metastases develop primarily via the hematogenous route, commonly involving the liver and lungs. GISTs also may invade adjacent organs directly or spread via peritoneal seeding. The 5-year survival rate for patients with malignant small bowel GISTs is approximately 25\% [18]. Patients with GISTs usually present with obscure gastrointestinal bleeding, which results from surface ulceration and necrosis [1, 5, 22] (fig. 2c). 
Other complications of GISTs include bowel obstruction, intussusception and tumor perforation [18]. The diagnosis of small bowel GISTs can be very difficult [22]. On barium studies, submucosal GISTs appear as smooth, round filling defects that may or may not ulcerate. Subserosal GISTs are extrinsic or exocentric masses that displace adjacent bowel loops. Intraluminal GISTs may be hypervascular and cause hemorrhage and ulceration. On CT, GISTs present as a mass that can be smooth, irregular or lobulated in appearance [18]. Pathology assessment should include immunohistochemical staining for KIT and possibly DOG1 expression, and mutational analysis can have prognostic and predictive value for certain patients [23].

\section{Therapy of GISTs}

The management of patients with GISTs typically involves a combination of surgical, pathologic and pharmacologic interventions. Nevertheless, surgery remains the standard of care for patients with localized GISTs [23, 24]. R0 resection is the goal for patients with localized or potentially resectable tumors, which often can be accomplished by laparoscopic resection, even for larger tumors. However, adjuvant imatinib can delay recurrence in some of these patients. In patients with advanced or metastatic disease, the standard of care is imatinib and surgery of residual masses ('debulking'). Cytoreductive surgery may have value for patients with recurrent or metastatic GISTs who exhibit stable disease or respond to tyrosine kinase inhibitor therapy. Preoperative imatinib is an emerging treatment option for patients who require cytoreductive therapy. Sunitinib is a standard second-line therapy [25]. It is important to emphasize that although tyrosine kinase inhibitors substantially improve survival in advanced disease, secondary drug resistance is common.

\section{Disclosure Statement}

None of the authors has any conflicts of interest related to this article/work to declare.

\section{References}

1 Zureikat AH, Heller MT, Zeh HJ III: Cancer of the small intestine; in DeVita VT Jr, Lawrence TS, Rosenberg SA (eds): Cancer: Principles and Practice of Oncology, ed 9. Philadelphia, Lippincott Williams \& Wilkins, 2011, pp 1048-1059.

2 Aparicio T, Zaanan A, Svrcek M, Laurent-Puig P, Carrere N, Manfredi S, Locher C, Afchain P: Small bowel adenocarcinoma: epidemiology, risk factors, diagnosis and treatment. Dig Liver Dis DOI: 10.1016/j.dld.2013.04.013.

-3 Haselkorn T, Whittemore AS, Lilienfeld DE: Incidence of small bowel cancer in the United States and worldwide: geographic, temporal, and racial differences. Cancer Causes Control 2005;16:781-787.

-4 Severson RK, Schenk M, Gurney JG, Weiss LK, Demers RY: Increasing incidence of adenocarcinomas and carcinoid tumors of the small intestine in adults. Cancer Epidemiol Biomarkers Prev 1996;5:81-84.

-5 Fry LC, Bellutti M, Neumann H, Malfertheiner P, Mönkemüller K: Incidence of bleeding lesions within reach of conventional upper and lower endoscopes in patients undergoing double-balloon enteroscopy for obscure gastrointestinal bleeding. Aliment Pharmacol Ther 2009;29:342-349.

-6 van Tuyl SA, van Noorden JT, Timmer R, Stolk MF, Kuipers EJ, Taal BG: Detection of small-bowel neuroendocrine tumors by video capsule endoscopy. Gastrointest Endosc 2006;64:66-72.

7 Achour J, Serraj I, Amrani L, Amrani N: Small bowel tumors: what is the contribution of video capsule endoscopy? Clin Res Hepatol Gastroenterol 2012;36:222-226.

8 Almeida N, Figueiredo P, Lopes S, Gouveia H, Leitão MC: Double-balloon enteroscopy and small bowel tumors: a South-European single-center experience. Dig Dis Sci 2009;54:1520-1524.

-9 Zouhairi ME, Venner A, Charabaty A, Pishvaian MJ: Small bowel adenocarcinoma. Curr Treat Options Oncol 2008;9:388-399.

10 Piscaglia AC, Campanale M, Gasbarrini G: Small bowel nonendocrine neoplasms: current concepts and novel perspectives. Eur Rev Med Pharmacol Sci 2010;14:320-326.

11 Fry LC, Neumann H, Kuester D, Kuhn R, Bellutti M, Malfertheiner P, Mönkemüller K: Small bowel polyps and tumours: endoscopic detection and treatment by double-balloon enteroscopy. Aliment Pharmacol Ther 2009; 29:135-142. 
12 Mönkemüller K, Fry LC, Neumann H: Endoscopic management of premalignant, early and advanced malignancies of the small bowel; in Deutsch JC, Banks MR (eds): Gastrointestinal Endoscopy in the Cancer Patient. Oxford, Wiley-Blackwell, 2013, pp 114-120.

13 Saurin JC, Gutknecht C, Napoleon B, Chavaillon A, Ecochard R, Scoazec JY, Ponchon T, Chayvialle JA: Surveillance of duodenal adenomas in familial adenomatous polyposis reveals high cumulative risk of advanced disease. J Clin Oncol 2004;22:493-498.

14 Mönkemüller K, Fry LC, Ebert M, Bellutti M, Venerito M, Knippig C, Rickes S, Muschke P, Röcken C, Malfertheiner P: Feasibility of double-balloon enteroscopy-assisted chromoendoscopy of the small bowel in patients with familial adenomatous polyposis. Endoscopy 2007;39:52-57.

15 Bellutti M, Fry LC, Schmitt J, Seemann M, Klose S, Malfertheiner P, Mönkemüller K: Detection of neuroendocrine tumors of the small bowel by double balloon enteroscopy. Dig Dis Sci 2009;54:1050-1058.

$\checkmark 16$ Demirkan BH, Eriksson B: Systemic treatment of neuroendocrine tumors with hepatic metastases. Turk J Gastroenterol 2012;23:427-437.

-17 Trikudanathan G, Dasanu CA: Evolving pharmacotherapeutic strategies for small bowel adenocarcinoma. Expert Opin Pharmacother 2010;11:1695-1704.

18 Gore RM, Mehta UK, Berlin JW, Rao V, Newmark GM: Diagnosis and staging of small bowel tumours. Cancer Imaging 2006;6:209-212.

19 Jovanovic I, Fry LC, Mönkemüller K: Small-bowel adenocarcinoma. Clin Gastroenterol Hepatol 2011; 9:A28.

20 Overman MJ, Kopetz S, Wen S, Hoff PM, Fogelman D, Morris J, Abbruzzese JL, Ajani JA, Wolff RA: Chemotherapy with 5 -fluorouracil and a platinum compound improves outcomes in metastatic small bowel adenocarcinoma. Cancer 2008;113:2038-2045.

-21 Overman MJ, Kopetz S, Lin E, Abbruzzese JL, Wolff RA: Is there a role for adjuvant therapy in resected adenocarcinoma of the small intestine. Acta Oncol 2010;49:474-479.

-22 Jovanovic I, Krivokapic Z, Menkovic N, Krstic M, Mönkemüler K: Ineffectiveness of capsule endoscopy and total double-balloon enteroscopy to elicit the cause of obscure overt gastrointestinal bleeding: think GIST! Endoscopy 2011;43(suppl 2 UCTN):E91-E92.

23 Joensuu H, Hohenberger P, Corless CL: Gastrointestinal stromal tumour. Lancet DOI: 10.1016/S01406736(13)60106-3.

24 Pisters PW, Patel SR: Gastrointestinal stromal tumors: current management. J Surg Oncol 2010;102:530-538.

25 Mullady DK, Tan BR: A multidisciplinary approach to the diagnosis and treatment of gastrointestinal stromal tumor. J Clin Gastroenterol 2013;47:578-585. 